ORIGINAL ARTICLE

\section{A Randomized Trial of Tenecteplase versus Alteplase for Acute Ischemic Stroke}

\author{
Mark Parsons, M.D., Neil Spratt, M.D., Andrew Bivard, B.Sc., \\ Bruce Campbell, M.D., Kong Chung, M.D., Ferdinand Miteff, M.D., \\ Bill O'Brien, M.D., Christopher Bladin, M.D., Patrick McElduff, Ph.D., \\ Chris Allen, M.D., Grant Bateman, M.D., Geoffrey Donnan, M.D., \\ Stephen Davis, M.D., and Christopher Levi, M.D.
}

\section{BACKGROUND}

Intravenous alteplase is the only approved treatment for acute ischemic stroke. Tenecteplase, a genetically engineered mutant tissue plasminogen activator, is an alternative thrombolytic agent.

\section{METHODS}

In this phase $2 \mathrm{~B}$ trial, we randomly assigned 75 patients to receive alteplase $(0.9 \mathrm{mg}$ per kilogram of body weight) or tenecteplase ( $0.1 \mathrm{mg}$ per kilogram or $0.25 \mathrm{mg}$ per kilogram) less than 6 hours after the onset of ischemic stroke. To favor the selection of patients most likely to benefit from thrombolytic therapy, the eligibility criteria were a perfusion lesion at least $20 \%$ greater than the infarct core on computed tomographic (CT) perfusion imaging at baseline and an associated vessel occlusion on CT angiography. The coprimary end points were the proportion of the perfusion lesion that was reperfused at 24 hours on perfusion-weighted magnetic resonance imaging and the extent of clinical improvement at 24 hours as assessed on the National Institutes of Health Stroke Scale (NIHSS, a 42-point scale on which higher scores indicate more severe neurologic deficits).

\section{RESULTS}

The three treatment groups each comprised 25 patients. The mean $( \pm$ SD) NIHSS score at baseline for all patients was $14.4 \pm 2.6$, and the time to treatment was $2.9 \pm 0.8$ hours. Together, the two tenecteplase groups had greater reperfusion $(\mathrm{P}=0.004)$ and clinical improvement $(\mathrm{P}<0.001)$ at 24 hours than the alteplase group. There were no significant between-group differences in intracranial bleeding or other serious adverse events. The higher dose of tenecteplase ( $0.25 \mathrm{mg}$ per kilogram) was superior to the lower dose and to alteplase for all efficacy outcomes, including absence of serious disability at 90 days (in $72 \%$ of patients, vs. $40 \%$ with alteplase; $\mathrm{P}=0.02$ ).

\section{CONCLUSIONS}

Tenecteplase was associated with significantly better reperfusion and clinical outcomes than alteplase in patients with stroke who were selected on the basis of CT perfusion imaging. (Funded by the Australian National Health and Medical Research Council; Australia New Zealand Clinical Trials Registry number, ACTRN12608000466347.)
From the Departments of Neurology (M.P., N.S., A.B., K.C., F.M., B.O., C.L.), Clinical Research Design, Information Technology, and Statistical Support (P.M.), and Radiology (C.A., G.B.), John Hunter Hospital-Hunter Medical Research Institute, University of Newcastle, Newcastle, NSW; the Department of Neurology, Royal Melbourne Hospital (B.C., S.D.), and Florey Neuroscience Institutes (G.D.), University of Melbourne, Melbourne, VIC; and the Department of Neurosciences, Box Hill Hospital-Eastern Health, Monash University (C.B.), Melbourne, VIC - all in Australia. Address reprint requests to Dr. Parsons at the Department of Neurology, John Hunter Hospital, Locked Bag No. 1, Hunter Region Mail Centre, Newcastle, NSW 2310, Australia, or at mark.parsons@hnehealth.nsw .gov.au.

N Engl J Med 2012;366:1099-107. Copyright (C) 2012 Massachusetts Medical Society. 
T HROMBOLYTIC TREATMENT WITH ALTEplase, a recombinant tissue plasminogen activator, for acute ischemic stroke is of proven benefit. ${ }^{1}$ However, alteplase is far from ideal, with incomplete and often delayed reperfusion in many patients. ${ }^{2}$ Tenecteplase, a genetically engineered mutant tissue plasminogen activator, has some pharmacokinetic advantages over alteplase. ${ }^{3} \mathrm{~A}$ balance between efficacy and risk of bleeding in the treatment of stroke appears to be achieved at a lower dose of tenecteplase than the dose used for myocardial infarction. ${ }^{4} \mathrm{~A}$ recent doseranging study of tenecteplase involving patients with acute ischemic stroke, which used standard clinical selection criteria, showed that a dose of $0.4 \mathrm{mg}$ per kilogram of body weight was associated with excess intracranial hemorrhage. The trial was stopped prematurely, owing to slow enrollment, with no difference shown between doses of $0.1 \mathrm{mg}$ per kilogram and $0.25 \mathrm{mg}$ per kilogram. ${ }^{5}$

In a nonrandomized pilot trial, we found that patients who received tenecteplase at a dose of $0.1 \mathrm{mg}$ per kilogram had superior outcomes on imaging (reperfusion and infarct growth) and greater early clinical improvement than patients who received alteplase at a dose of $0.9 \mathrm{mg}$ per kilogram. ${ }^{6}$ However, only the patients who received tenecteplase were treated on the basis of computed tomographic (CT) perfusion and angiographic imaging. We conducted a phase $2 \mathrm{~B}$, randomized trial to compare the standard dose of alteplase with two different doses of tenecteplase. CT perfusion and angiographic imaging was used to select patients who would be the most likely to benefit from early reperfusion (i.e., patients with large-vessel occlusion and a large perfusion lesion in the absence of a large infarct core).

METHODS

STUDY DESIGN AND PATIENTS

In this randomized, open-label, blinded trial, patients with acute ischemic stroke underwent CT perfusion and angiographic imaging before receiving treatment with intravenous tenecteplase or alteplase, which was administered within 6 hours after the onset of the stroke. Patients underwent magnetic resonance imaging (MRI) at 24 hours and at 90 days for assessment of imaging outcomes. The trial was performed between 2008 and 2011 in three large stroke centers in Australia. The study design is shown in the Supplementary Appendix, which is available with the full text of this article at NEJM.org.

We enrolled patients with first-ever hemispheric ischemic stroke who were 18 years of age or older, had a score greater than 4 on the National Institutes of Health Stroke Scale (NIHSS; a 42-point scale that quantifies neurologic deficits in 11 categories, with higher scores indicating more severe deficits), and a premorbid score of 2 or less on the modified Rankin scale (which ranges from 0 to 6 , with 0 indicating no symptoms and 6 indicating death) (Table 1). Exclusion criteria were standard contraindications to alteplase. ${ }^{7,8}$

In addition, we used specific selection criteria for this trial that were based on the results of CT imaging. ${ }^{6}$ The CT angiographic criterion was the presence of intracranial occlusion in the anterior cerebral, middle cerebral, or posterior cerebral artery. Patients with internal-carotid-artery and vertebrobasilar occlusions were excluded. The CT perfusion criterion was a hemispheric perfusion lesion on transit-time maps that was at least $20 \%$ greater than the infarct-core lesion, with a volume of at least $20 \mathrm{ml}$. The infarct-core lesion on CT perfusion maps of cerebral blood volume had to be less than one third the territory of the middle cerebral artery or less than one half the territory of the anterior cerebral or posterior cerebral artery.

The review of CT images was performed at a clinical imaging workstation with the use of instrument-specific proprietary software. The on-console image analysis was performed by the treating stroke neurologists, all of whom had experience in the evaluation of CT perfusion and angiographic imaging. ${ }^{9}$

Patients were randomly assigned in a 1:1:1 ratio to the standard dose of alteplase $(0.9 \mathrm{mg}$ per kilogram, the first $10 \%$ administered as an initial bolus and the remainder over a 1-hour period, with a maximum dose of $90 \mathrm{mg}$ ) or to tenecteplase ( $0.1 \mathrm{mg}$ per kilogram, administered as a single bolus, with a maximum dose of $10 \mathrm{mg}$; or $0.25 \mathrm{mg}$ per kilogram, administered as a single bolus, with a maximum dose of $25 \mathrm{mg}$ ). A central block randomization was performed by the Centre for Clinical Epidemiology and Biostatistics, University of Newcastle, in blocks of 15 to allow the data and safety monitoring board to review blinded safety data after the recruitment of every 15 patients. Randomization was performed by means of a central telephone service. The treating clinician was aware of the treatment assignments. 


\begin{tabular}{|c|c|c|c|}
\hline \multirow[t]{2}{*}{ Characteristic } & \multirow[t]{2}{*}{ Alteplase $(\mathrm{N}=25)$} & \multicolumn{2}{|c|}{ Tenecteplase } \\
\hline & & $\begin{array}{c}0.1 \mathrm{mg} / \mathrm{kg} \\
(\mathrm{N}=25)\end{array}$ & $\begin{array}{c}0.25 \mathrm{mg} / \mathrm{kg} \\
(\mathrm{N}=25)\end{array}$ \\
\hline \multicolumn{4}{|l|}{ Clinical } \\
\hline Age $-y r$ & $70 \pm 8.4$ & $72 \pm 6.9$ & $68 \pm 9.4$ \\
\hline Male sex — no. (\%) & $12(48)$ & $13(52)$ & $13(52)$ \\
\hline Hypertension — no. (\%) & $15(60)$ & $16(64)$ & $16(64)$ \\
\hline Diabetes mellitus — no. (\%) & $1(4)$ & $8(32)$ & $6(24)$ \\
\hline Blood glucose $-\mathrm{mmol} / \mathrm{liter}$ & $6.4 \pm 1.1$ & $7.1 \pm 2.0$ & $7.3 \pm 1.8$ \\
\hline Hyperlipidemia - no. (\%) & $9(36)$ & $13(52)$ & $15(60)$ \\
\hline Atrial fibrillation - no. (\%) & $6(24)$ & $9(36)$ & $13(52)$ \\
\hline Current smoking - no. (\%) & $1(4)$ & $9(36)$ & $5(20)$ \\
\hline \multicolumn{4}{|l|}{ Current medications - no. (\%) } \\
\hline Antiplatelet agent & $11(44)$ & $11(44)$ & $12(48)$ \\
\hline Anticoagulant & $1(4)$ & $1(4)$ & $1(4)$ \\
\hline NIHSS score† & $14.0 \pm 2.3$ & $14.5 \pm 2.3$ & $14.6 \pm 2.3$ \\
\hline Time to treatment $-\mathrm{hr}$ & $2.7 \pm 0.8$ & $3.1 \pm 0.9$ & $3.0 \pm 0.7$ \\
\hline \multicolumn{4}{|l|}{ Imaging } \\
\hline \multicolumn{4}{|l|}{ Volume of infarct core $-\mathrm{ml}$} \\
\hline Median & 13 & 8 & 11 \\
\hline Interquartile range & $2-41$ & $1-25$ & $1-35$ \\
\hline \multicolumn{4}{|l|}{ Volume of perfusion lesion - $\mathrm{ml}$} \\
\hline Median & 76 & 80 & 79 \\
\hline Interquartile range & 21-185 & 22-199 & $31-147$ \\
\hline \multicolumn{4}{|l|}{ Occlusion site - no. (\%) } \\
\hline Anterior cerebral artery & 0 & 0 & $1(4)$ \\
\hline Proximal section of first segment of middle cerebral artery & $11(44)$ & $6(24)$ & $8(32)$ \\
\hline Midsection of first segment of middle cerebral artery & $2(8)$ & $4(16)$ & $4(16)$ \\
\hline Distal section of first segment of middle cerebral artery & $5(20)$ & $10(40)$ & $7(28)$ \\
\hline Second segment of middle cerebral artery & $4(16)$ & $2(8)$ & $4(16)$ \\
\hline Posterior cerebral artery & $1(4)$ & $1(4)$ & $1(4)$ \\
\hline Terminal internal carotid artery & 0 & $1(4)$ & 0 \\
\hline None & $2(8)$ & $1(4)$ & 0 \\
\hline
\end{tabular}

* Plus-minus values are means \pm SD. Lesion volumes are rounded to the nearest milliliter. There were no significant differences between the alteplase group and the pooled tenecteplase groups except that the alteplase group had fewer people with diabetes $(P=0.01)$, fewer smokers $(P=0.01)$, and a lower mean blood glucose level $(P=0.05)$. To convert the values for glucose to milligrams per deciliter, divide by 0.05551 . There were no significant differences in baseline characteristics between the two tenecteplase groups.

$\uparrow$ Scores on the National Institutes of Health Stroke Scale (NIHSS) range from 0 to 42; higher scores indicate more severe neurologic deficits.

\section{STUDY OVERSIGHT}

The steering committee, comprising five of the authors, designed and oversaw the trial and vouches for the completeness and accuracy of the data and the analysis. The data analysis was undertaken by four of the authors. One of the authors, a biostatistician, was responsible for the unblinding of the treatment assignments after the database was cleaned and locked, and performed the prespecified statistical analyses. Boehringer Ingelheim sup- 
plied tenecteplase at a discount but was not involved in the study design, study conduct, data management, data analysis, or manuscript preparation. Alteplase was supplied by the treating hospital, because it is the standard treatment.

Written informed consent was provided by the patients or their health care proxies. The study protocol (available at NEJM.org) and informedconsent procedures were approved by the institutional review board at each participating center.

\section{PROCEDURES}

CT perfusion and angiographic imaging was performed with multidetector scanners (16- or 64-slice) before randomization. Whole-brain CT imaging, without the administration of contrast material was followed by CT perfusion imaging, comprising two 60 -second series, each performed after an intravenous bolus of $40 \mathrm{ml}$ of iodinated contrast agent at a concentration of $370 \mathrm{mg}$ per milliliter, followed by a $40-\mathrm{ml}$ saline flush at a rate of $6 \mathrm{ml}$ per second..$^{10}$ Each perfusion series covered an axial section of 24 to $40 \mathrm{~mm}$, acquired as adjacent slices of 5 to $8 \mathrm{~mm} .{ }^{11} \mathrm{CT}$ angiography was performed after CT perfusion imaging, with images acquired from the carotid bifurcation to the top of the lateral ventricles.

MRI was performed with the use of 1.5-T scanners. Standardized sequences were obtained 24 hours after treatment and included an axial gradient-echo $\mathrm{T}_{2}$-weighted series, a diffusionweighted echoplanar spin-echo sequence, time-offlight magnetic resonance angiography, perfusionweighted imaging, and a fluid-attenuated inversion recovery (FLAIR) sequence. ${ }^{12,13}$ At 90 days after treatment, FLAIR imaging was repeated to measure the final infarct volume.

\section{OUTCOMES}

The coprimary outcomes were the percentage of the perfusion lesion that was reperfused 24 hours after treatment, as assessed on perfusion-weighted MRI, and the extent of clinical improvement at 24 hours, as measured by the change on the NIHSS score from before treatment to 24 hours after treatment. ${ }^{6,14}$ Secondary imaging efficacy outcomes were the extent of infarct growth at 24 hours and at 90 days and vessel recanalization at 24 hours. ${ }^{15,16}$

Secondary clinical efficacy outcomes were major neurologic improvement at 24 hours (defined as a reduction from baseline of 8 or more points on the NIHSS), excellent recovery at 90 days (de- fined as a score of 0 or 1 on the modified Rankin scale), and excellent or good recovery at 90 days (defined as a score of 0 to 2). ${ }^{1,7,17,18}$ These scores on the modified Rankin scale have been used as primary outcomes in all major phase 3 trials of thrombolytic agents: a score of 0 indicates no symptoms, 1 minor symptoms but no clinically significant disability, and 2 slight disability. 1,7,19 Secondary imaging safety outcomes were the occurrence of large parenchymal hematoma ( $>30 \%$ of the infarct volume), parenchymal hematoma of any size, and symptomatic intracranial hemorrhage. Secondary clinical safety outcomes were poor outcome (i.e., severe disability) or death at 90 days, defined as a score of 5 or 6 , respectively, on the modified Rankin scale. See the Supplementary Appendix for details of outcomes.

All analyses of imaging outcomes were performed centrally on de-identified data. Assessors were unaware of the treatment assignments and clinical information. CT and MRI data were analyzed with the use of commercial software (MIStar, Apollo Medical Imaging). ${ }^{20}$ See the Supplementary Appendix for details of the imaging outcomes.

Scoring on the NIHSS was performed immediately before baseline imaging and at 24 hours. Scoring on the modified Rankin scale was performed at baseline and on day 90. The clinical assessments at 24 hours and on day 90 were performed by a trained observer who was not involved in the patients' clinical care and who was unaware of the treatment assignments. The observer calculated the patients' scores on the NIHSS or the modified Rankin scale in the presence of a study coordinator, who ensured that the observer remained unaware of the treatment assignments.

\section{STATISTICAL ANALYSIS}

Before the completion of the study, a biostatistician developed the statistical analysis plan to test the prespecified trial hypotheses defined in the protocol. The primary hypothesis was that a comparison between the alteplase group and the pooled tenecteplase groups would show the superiority of tenecteplase with respect to one or both coprimary outcomes (percent reperfusion and change in the NIHSS score at 24 hours). An alpha level of 0.025 was prespecified for each of the two primary end points. We calculated the sample size for the current study on the basis of our pilot study, with power set at $80 \%$ and an assumption of superiority with respect to one of the two coprimary 
outcomes, at an alpha level of 0.025 , in the pooled tenecteplase groups.

Primary hypotheses were tested by means of an unadjusted Student's t-test of means. This analysis was repeated after adjustment for potential confounding baseline variables that differed between the alteplase and pooled tenecteplase groups $(\mathrm{P}<0.10)$ (Table 1$)$. Secondary outcomes with a nonparametric distribution were tested with the use of the Wilcoxon rank-sum test, and categorical variables were compared with the use of the chi-square test of proportions or Fisher's exact test. In the event of support for the primary hypothesis, the protocol specified an analysis to compare efficacy and safety outcomes between the two tenecteplase groups and between each of the tenecteplase groups and the alteplase group.

After the initial trial registration but before the completion of the study, the trial end points were modified, as informed by several studies. ${ }^{6,13,17,20}$ This led to the modification of the reperfusion primary outcome from absolute volume change to proportional change. ${ }^{6,13}$ Infarct growth replaced "mismatch salvage" (i.e., the volume of mismatch tissue on CT perfusion imaging at baseline that did not progress to infarction on follow-up MRI) as a secondary outcome, and the intracranialhemorrhage outcomes were expanded (see the Supplementary Appendix). The prespecified statistical analysis plan was based on these slightly modified end points. However, after the study and the per-protocol prespecified analyses were completed, we performed post hoc analyses of the original end points (see the Supplementary Appendix).

\section{RESULTS}

\section{STUDY PATIENTS}

Figure 1 shows the screening profile of the trial. Between 2008 and 2011, a total of 2768 patients presenting with strokelike symptoms underwent screening within 6 hours after symptom onset; of these patients, $75(3 \%)$ were enrolled in the study. The majority of patients screened (2164 [78\%]) were not eligible for intravenous thrombolysis on the basis of standard clinical exclusions (Fig. 1). This left 604 patients (22\%) potentially eligible for intravenous thrombolysis, 127 (5\%) of whom met the additional CT selection criteria. The major reasons for exclusion that were based on these criteria were internal-carotid-artery occlusion (116 pa-

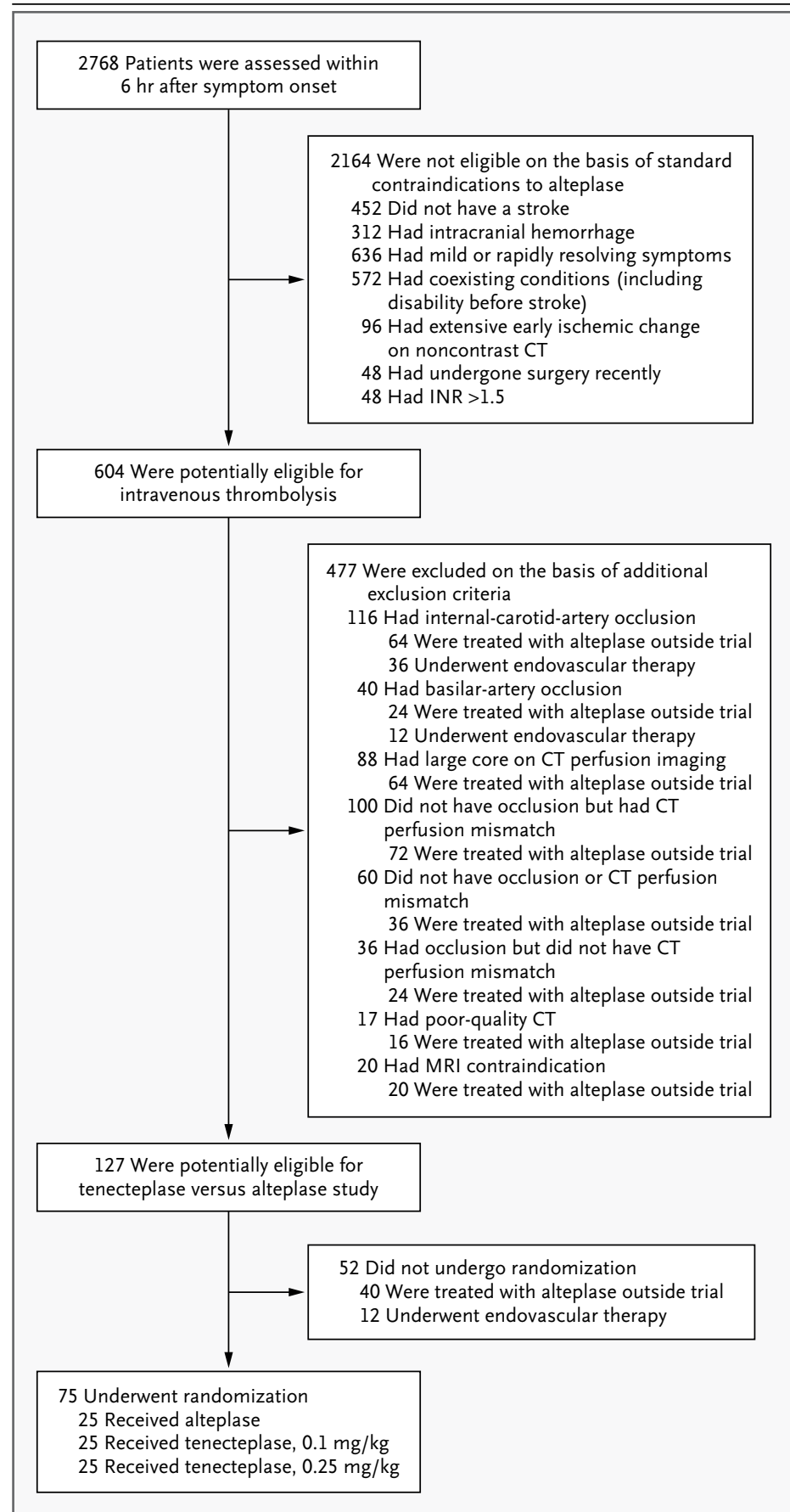

Figure 1. Study Enrollment.

Of the 2768 patients who were screened for participation in the study, 75 underwent randomization to the three treatment groups. INR denotes international normalized ratio.

tients [4\%]), a large infarct core on CT perfusion imaging (88 [3\%]), and an absence of intracranial vessel occlusion (160 [6\%]). Most of the patients 
who were eligible for intravenous thrombolysis but did not meet the CT criteria for this study received open-label alteplase (320 of 477 patients [67\%]); some received endovascular therapy (48 of 477 [10\%]). Of the 127 patients who met the additional CT selection criteria, 75 were enrolled. The reasons that 52 eligible patients were not enrolled were the patient's or physician's preference for open-label alteplase treatment (40 patients) and a decision by the treating clinician to proceed with endovascular therapy (12 patients).

Of the 75 enrolled patients, 25 were randomly assigned to each of the three treatment groups. Table 1 shows the baseline characteristics of these patients. The mean $( \pm S D)$ NIHSS score for all patients in the trial was relatively high at 14.4 2.6. Most baseline clinical characteristics were well matched among the three groups, but the alteplase group included fewer persons who smoked $(\mathrm{P}=0.01)$; this group also included fewer persons with diabetes $(\mathrm{P}=0.01)$, which was reflected in a lower blood glucose level at baseline in the alteplase group $(\mathrm{P}=0.05)$. Patients in the alteplase group received treatment at a mean of 2.7 hours, as compared with 3.1 hours for those in the pooled tenecteplase groups $(\mathrm{P}=0.06)$. Only 3 patients were treated after 4.5 hours. There were no significant differences in the imaging characteristics at baseline among the treatment groups. There were no significant differences in the site of occlusion at baseline, but on central blinded review of the CT angiographic data, the vessel-occlusion criteria were not met in 4 patients (Table 1). One patient had an occlusion of the terminal internal carotid artery, and 3 patients ( 2 in the alteplase group) had no definite occlusion.

\section{EFFICACY}

There was a significant benefit associated with tenecteplase for both coprimary end points of the study (Table 2), with greater reperfusion $(\mathrm{P}=0.004)$ and greater clinical improvement $(\mathrm{P}<0.001)$ at 24 hours in the pooled tenecteplase groups than in the alteplase group. The magnitude and significance of the reperfusion and early clinical improvement in the pooled tenecteplase groups did not change after correction for imbalances at baseline (Table 2). Tenecteplase was also beneficial with respect to secondary outcomes. In the pooled tenecteplase groups, as compared with the alteplase group, infarct growth was reduced, and a higher proportion of patients had an excellent or good recovery (modified Rankin scale score of 0 to 2) at 90 days ( $72 \%$ vs. $44 \%, \mathrm{P}=0.02)$.

\section{SAFETY}

Seven patients died: 3 in the alteplase group $(12 \%), 3$ in the group that received the lower dose of tenecteplase (12\%), and 1 in the group that received the higher dose of tenecteplase (4\%). Two deaths in the alteplase group were due to massive hemispheric infarction, and one to symptomatic intracranial hemorrhage. In the lower-dose tenecteplase group, one death was due to symptomatic intracranial hemorrhage, one to aspiration pneumonia, and one to a late second stroke. The one death in the higher-dose tenecteplase group was due to multiple coexisting conditions (pneumonia, myocardial infarction, and acute-on-chronic renal failure). Two of the 50 patients in the tenecteplase group (4\%) had large parenchymal hematomas, as compared with 4 of the 25 in the alteplase group ( $16 \%, \mathrm{P}=0.09)$. Five of the 6 patients with large parenchymal hematomas also had symptomatic deterioration of 4 or more points on the NIHSS at 24 hours and poor outcome at 90 days (modified Rankin scale score of 5 or 6). Poor outcome at 90 days occurred in 7 patients in the alteplase group (28\%) and in $5(10 \%)$ in the pooled tenecteplase groups $(\mathrm{P}=0.09)$.

\section{DOSE-TIER ANALYSIS}

The higher dose of tenecteplase $(0.25 \mathrm{mg}$ per kilogram) was associated with improvement on all imaging efficacy outcomes, as compared with alteplase (see the Supplementary Appendix). In addition to an increased proportion of patients with early clinical improvement, the outcomes at 3 months were better in the higher-dose tenecteplase group; $72 \%$ of patients in this group had an excellent recovery (no clinically significant disability), as compared with $40 \%$ of those in the alteplase group $(\mathrm{P}=0.02)$. There were no more adverse outcomes with either dose of tenecteplase than with alteplase (see the Supplementary Appendix). The patients who received the lower dose of tenecteplase had greater clinical improvement at 24 hours than did the patients who received alteplase $(\mathrm{P}=0.04)$, but other efficacy outcomes were equivalent between the two groups.

There was an efficacy dose response between the two tenecteplase dose tiers, including higher 
Table 2. Study Outcomes in the Alteplase and Pooled Tenecteplase Groups.*

\begin{tabular}{|c|c|c|c|}
\hline Outcome & $\begin{array}{l}\text { Alteplase } \\
(\mathrm{N}=\mathbf{2 5})\end{array}$ & $\begin{array}{l}\text { Tenecteplase } \\
(\mathrm{N}=50)\end{array}$ & P Value \\
\hline \multicolumn{4}{|l|}{ Primary imaging efficacy outcome } \\
\hline Reperfusion at $24 \mathrm{hr}-\% \dagger$ & $55.4 \pm 38.7$ & $79.3 \pm 28.8$ & 0.004 \\
\hline \multicolumn{4}{|l|}{ Primary clinical efficacy outcome } \\
\hline Improvement in NIHSS score between baseline and $24 \mathrm{hrt}$ & $3.0 \pm 6.3$ & $8.0 \pm 5.5$ & $<0.001$ \\
\hline \multicolumn{4}{|l|}{ Secondary imaging efficacy outcome } \\
\hline \multicolumn{4}{|l|}{ Infarct growth at $24 \mathrm{hr}-\mathrm{ml}$} \\
\hline Median & 14 & 3 & 0.04 \\
\hline Interquartile range & 0 to 144 & -1 to 121 & \\
\hline \multicolumn{4}{|l|}{ Infarct growth at 90 days $-\mathrm{ml}$} \\
\hline Median & 12 & 2 & 0.01 \\
\hline Interquartile range & -1 to 113 & -2 to 133 & \\
\hline Complete recanalization at $24 \mathrm{hr}-$ no./total no. (\%) & $8 / 22(36)$ & $28 / 48(58)$ & 0.09 \\
\hline Complete or partial recanalization at $24 \mathrm{hr}$ - no./total no. (\%) & $15 / 22(68)$ & $42 / 48(88)$ & 0.05 \\
\hline \multicolumn{4}{|l|}{ Secondary imaging safety outcome } \\
\hline Large parenchymal hematoma - no. (\%) & $4(16)$ & $2(4)$ & 0.09 \\
\hline Any parenchymal hematoma — no. (\%) & $5(20)$ & $3(6)$ & 0.11 \\
\hline Symptomatic intracranial hematoma - no. (\%) $\int$ & $3(12)$ & $2(4)$ & 0.33 \\
\hline \multicolumn{4}{|l|}{ Secondary clinical efficacy outcome } \\
\hline $\begin{array}{l}\text { Major neurologic improvement at } 24 \mathrm{hr} \text {, reduction of } \geq 8 \text { in NIHSS } \\
\text { score - no. (\%) }\end{array}$ & $9(36)$ & $32(64)$ & 0.02 \\
\hline Excellent recovery at 90 days — no. (\%) 9 & $10(40)$ & $27(54)$ & 0.25 \\
\hline Excellent or good recovery at 90 days - no. (\%) $\emptyset$ & $11(44)$ & $36(72)$ & 0.02 \\
\hline \multicolumn{4}{|l|}{ Secondary clinical safety outcome } \\
\hline Poor outcome at 90 days - no. (\%) 9 & $7(28)$ & $5(10)$ & 0.09 \\
\hline Death — no. (\%) & $3(12)$ & $4(8)$ & 0.68 \\
\hline \multicolumn{4}{|l|}{ Post hoc secondary imaging outcome } \\
\hline Volume reperfusion at $24 \mathrm{hr}-\mathrm{ml}$ & $38.2 \pm 30.9$ & $69.3 \pm 34.6$ & 0.002 \\
\hline Mismatch salvage at $24 \mathrm{hr}-\mathrm{ml} \|$ & $55.8 \pm 39.9$ & $80.7 \pm 26.5$ & 0.002 \\
\hline Mismatch salvage at 90 days $-\mathrm{ml} \|$ & $58.3 \pm 38.9$ & $83.3 \pm 26.2$ & 0.003 \\
\hline
\end{tabular}

* Plus-minus values are means \pm SD. Lesion volumes are rounded to the nearest milliliter. Two patients did not undergo $\mathrm{MRI}$ at 24 hours owing to clinical deterioration (one in the lower-dose tenecteplase group and one in the alteplase group) and were not included in the analysis of the primary reperfusion outcome (or other imaging-based efficacy outcomes). Five patients died before day 90 and were not included in the analysis of infarct growth at day 90 . Five patients were not included in the analysis of recanalization outcomes: the two who did not undergo MRI at 24 hours and the three without occlusions at baseline.

$\dagger$ The mean percentage of reperfusion remained significant $(P=0.003)$ after adjustment for the baseline variables of status with respect to diabetes and smoking, blood glucose level, and time to treatment.

$¥$ The mean change in the NIHSS score at 24 hours remained significant $(P=0.001)$ after adjustment for the baseline variables of status with respect to diabetes and smoking, blood glucose level, and time to treatment.

$\int$ This outcome was defined as a large parenchymal hematoma and clinical worsening (an increase in the NIHSS score of 4 or more points). ${ }^{17,21,22}$

ๆ Recovery was assessed with the modified Rankin scale, which ranges from 0 to 6 , with higher scores indicating greater disability. Excellent recovery was defined as a score of 0 or 1 , excellent or good recovery as 0 to 2 , and a poor outcome as 5 or 6 .

|| The mismatch salvage was defined as the volume of mismatch on computed tomographic perfusion imaging at baseline that did not progress to infarction. 

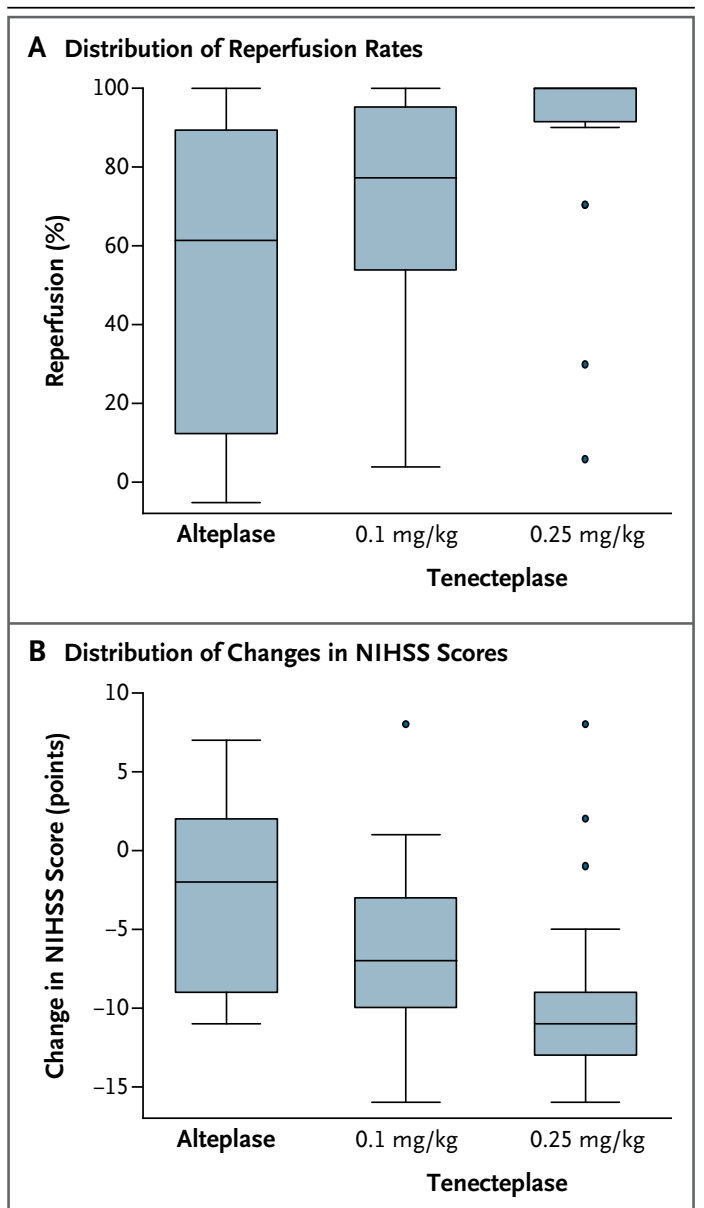

Figure 2. Box Plots for the Primary End Points for the Individual Dose Tiers.

Panel A shows the reperfusion rates at 24 hours, and Panel B shows the changes in the NIHSS score at 24 hours. Negative values for the change in the NIHSS score indicate improvement. The horizontal line inside each box indicates the median, the top and bottom of the box indicate the interquartile range, the I bars indicate the 5th and 95th percentiles, and the circles indicate outliers. The median value for tenecteplase at a dose of $0.25 \mathrm{mg}$ per kilogram was $100 \%$, which overlaps with the 75th percentile (top of box)

reperfusion and recanalization rates at the higher dose (Fig. 2, and the Supplementary Appendix). This translated into greater clinical improvement at 24 hours and an increase in the number of patients with excellent recovery (no clinically significant disability) at 90 days $(\mathrm{P}=0.01)$.

\section{DISCUSSION}

In this study, using CT perfusion and angiographic imaging to select patients for thrombolytic treat- ment of acute ischemic stroke, we found that tenecteplase was superior to alteplase with respect to the coprimary end points of reperfusion and clinical improvement at 24 hours. It is conventional in studies of acute stroke to use a primary outcome that assesses disability status at 90 days. However, reperfusion needs to be measured at a shorter interval after early reperfusion treatment in order to assess biologic efficacy, so we chose a primary clinical outcome that was most likely to be sensitive to early reperfusion. A longer-term clinical benefit was also seen, particularly with the higher dose of tenecteplase. The improved reperfusion and clinical response with tenecteplase did not come at a cost of increased intracranial hemorrhage. There was also a dose-response relationship, with the higher dose of tenecteplase being superior to both the lower dose of tenecteplase and alteplase for all imaging and clinical efficacy outcomes. These positive findings are encouraging but preliminary, owing to the small size of the study.

The results of this study suggest that it is appropriate to proceed to a phase 3 trial of tenecteplase versus alteplase in the time window that is currently approved for stroke thrombolysis. We believe that the dose response seen in the current study provides sufficient justification to use tenecteplase at a dose of $0.25 \mathrm{mg}$ per kilogram as the comparator. However, for this phase $2 \mathrm{~B}$ trial, we used criteria based on CT perfusion and angiographic imaging to select patients for inclusion who would be most likely to have a clinical benefit from early and effective reperfusion. These selection criteria enhanced the power of the study to detect a difference in efficacy between tenecteplase and alteplase with the use of a relatively small sample. A large number of patients who were eligible for thrombolysis on the basis of standard clinical and noncontrast CT criteria were excluded from this trial because of these additional imaging selection requirements. We therefore cannot extrapolate our results to the majority of patients who are eligible for thrombolysis. A phase 3 study would be needed to determine whether the efficacy of tenecteplase extends to this broader population of patients.

Supported by a grant from the Australian National Health and Medical Research Council.

Dr. Parsons reports receiving advisory-board fees from Bayer Australia; Dr. Campbell, consultancy fees from Lundbeck, speaker payments from Boehringer Ingelheim Australia, and grant support from Cardiovascular Lipid Australia; Dr. Bladin, advisory-board fees from Bayer Australia; Dr. Donnan, consultancy fees from 
Boehringer Ingelheim Australia and Bayer Australia; Dr. Davis, consultancy fees from Orsan Technologies and Ever Pharma; and Dr. Levi, consultancy fees from Boehringer Ingelheim Australia. No other potential conflict of interest relevant to this article was reported.
Disclosure forms provided by the authors are available with the full text of this article at NEJM.org.

We thank the study coordinators at each of the centers: Michelle Russell (central coordinator, John Hunter Hospital), Gabriel Silver (Royal Melbourne Hospital), and Zofia Ross (Box Hill Hospital).

\section{REFERENCES}

1. Lees KR, Bluhmki E, von Kummer R, et al. Time to treatment with intravenous alteplase and outcome in stroke: an updated pooled analysis of ECASS, ATLANTIS, NINDS, and EPITHET trials. Lancet 2010; 375:1695-703.

2. Neumann-Haefelin $\mathrm{T}$, du Mesnil de Rochemont R, Fiebach JB, et al. Effect of incomplete (spontaneous and postthrombolytic) recanalization after middle cerebral artery occlusion: a magnetic resonance imaging study. Stroke 2004;35:109-14.

3. Tanswell P, Modi N, Combs D, Danays T. Pharmacokinetics and pharmacodynamics of tenecteplase in fibrinolytic therapy of acute myocardial infarction. Clin Pharmacokinet 2002;41:1229-45.

4. Haley EC Jr, Lyden PD, Johnston KC, Hemmen TM. A pilot dose-escalation safety study of tenecteplase in acute ischemic stroke. Stroke 2005;36:607-12.

5. Haley EC Jr, Thompson JL, Grotta JC et al. Phase IIB/III trial of tenecteplase in acute ischemic stroke: results of a prematurely terminated randomized clinical trial. Stroke 2010;41:707-11.

6. Parsons MW, Miteff F, Bateman GA, et al. Acute ischemic stroke: imagingguided tenecteplase treatment in an extended time window. Neurology 2009;72 915-21.

7. Hacke W, Kaste M, Bluhmki E, et al. Thrombolysis with alteplase 3 to 4.5 hours after acute ischemic stroke. N Engl J Med 2008;359:1317-29.

8. Hacke W, Kaste M, Fieschi C, et al. Intravenous thrombolysis with recombinant tissue plasminogen activator for acute hemispheric stroke: the European Cooperative Acute Stroke Study (ECASS). JAMA 1995;274:1017-25.

9. Parsons MW, Pepper EM, Chan V, et al. Perfusion computed tomography: prediction of final infarct extent and stroke outcome. Ann Neurol 2005;58:672-9.

10. Wintermark M, Albers GW, Alexandrov AV, et al. Acute stroke imaging research roadmap. Stroke 2008;39:1621-8. 11. Parsons MW, Pepper EM, Bateman GA, Wang Y, Levi CR. Identification of penumbra and infarct core on hyperacute non-contrast and perfusion CT. Neurology 2007;68:730-6.

12. Bivard A, McElduff P, Spratt N, Levi C, Parsons M. Defining the extent of irreversible brain ischemia using perfusion computed tomography. Cerebrovasc Dis 2011;31:238-45.

13. Davis SM, Donnan GA, Parsons MW, et al. Effects of alteplase beyond $3 \mathrm{~h}$ after stroke in the Echoplanar Imaging Thrombolytic Evaluation Trial (EPITHET): a placebo-controlled randomised trial. Lancet Neurol 2008;7:299-309.

14. Soares BP, Tong E, Hom J, et al. Reperfusion is a more accurate predictor of follow-up infarct volume than recanalization: a proof of concept using CT in acute ischemic stroke patients. Stroke 2010; 41(1):e34-e40.

15. De Silva DA, Fink JN, Christensen S, et al. Assessing reperfusion and recanalization as markers of clinical outcomes after intravenous thrombolysis in the Echoplanar Imaging Thrombolytic Evaluation Trial (EPITHET). Stroke 2009;40:2872-4.
16. Khatri P, Neff J, Broderick JP, Khoury JC, Carrozzella J, Tomsick T. Revascularization end points in stroke interventional trials: recanalization versus reperfusion in IMS-I. Stroke 2005;36:2400-3.

17. Ahmed N, Wahlgren N, Grond M, et al. Implementation and outcome of thrombolysis with alteplase 3-4.5 $\mathrm{h}$ after an acute stroke: an updated analysis from SITS-ISTR. Lancet Neurol 2010;9:866-74. 18. Brown DL, Johnston KC, Wagner DP, Haley EC Jr. Predicting major neurological improvement with intravenous recombinant tissue plasminogen activator treatment of stroke. Stroke 2004;35:147-50.

19. Hacke W, Kaste M, Fieschi C, et al. Randomised double-blind placebo-controlled trial of thrombolytic therapy with intravenous alteplase in acute ischaemic stroke (ECASS II). Lancet 1998;352:124551.

20. Bivard A, Spratt N, Levi C, Parsons M. Perfusion computer tomography: imaging and clinical validation in acute ischaemic stroke. Brain 2011;134:3408-16. 21. Wahlgren N, Ahmed N, Dávalos A, et al. Thrombolysis with alteplase 3-4.5 h after acute ischaemic stroke (SITS-ISTR): an observational study. Lancet 2008;372: 1303-9.

22. Simpson MA, Dewey HM, Churilov L, et al. Thrombolysis for acute stroke in Australia: outcomes from the Safe Implementation of Thrombolysis in Stroke registry (2002-2008). Med J Aust 2010;193: 439-43.

Copyright (c) 2012 Massachusetts Medical Society. 